\title{
CD44v7 ligation downregulates the inflammatory immune response in Crohn's disease patients by apoptosis induction in mononuclear cells from the lamina propria
}

\author{
U Hoffmann ${ }^{1}$, K Heilmann ${ }^{1}$, C Hayford ${ }^{1}$, A Stallmach ${ }^{2}$, U Wahnschaffe ${ }^{1}$, M Zeitz ${ }^{1}$, U Günthert ${ }^{3}$ and BM Wittig ${ }^{\star, 1}$
}

Deletion of exon CD44v7 abrogates experimental colitis by apoptosis induction in intestinal mononuclear cells. Here we show that CD44v7 expression was upregulated upon CD40 ligation in human mononuclear cells, and examined whether ligation of CD44v7 also affects activation and apoptosis in lamina propria mononuclear cells (LPMC) from Crohn's disease (CD) patients. Thirty five patients with chronic inflammatory bowel disease (IBD), fourteen controls and four patients with diverticulitis were evaluated. CD44v7 was upregulated predominantly in the inflamed mucosa of CD patients. Furthermore, incubation with an antiCD44v7 antibody induced apoptosis in LPMC isolated from inflamed mucosa of CD patients, but not from non-inflamed mucosa, from patients with ulcerative colitis (UC) or from normal controls. CD40 ligation and simultaneous incubation with anti-CD44v7 significantly downregulated CD80 in dendritic cells, thus inhibiting a critical second signal for naive T-cell activation. The apoptotic signal was mediated via the intrinsic mitochondrial pathway with decreased Bcl-2 and increased 7A6 (a mitochondrial membrane protein) expression. It was Fas independent and required caspases-3 and -9 activation. The process is highly specific for macrophage activation via CD40. These findings point to a novel mechanism of apoptosis induction in CD patients mediated by CD44v7 ligation.

Cell Death and Differentiation (2007) 14, 1542-1551; doi:10.1038/sj.cdd.4402153; published online 4 May 2007

The mucosal immune system is in a constant state of lowgrade activation restrained by well-regulated mechanisms that control the inflammatory force. ${ }^{1-3}$ This homeostasis is achieved by equilibrium between leukocyte activation and programmed cell death. In patients with Crohn's disease (CD), the apoptosis pathway of lamina propria lymphocytes was found to be dysregulated. ${ }^{4}$ It was suggested that $C D$ results from a defect in the control mechanisms of apoptosis leading to inappropriate T-cell accumulation and disease perpetuation. ${ }^{5,6}$ Consequently, induction of T-cell apoptosis has remarkable therapeutic effects in experimental colitis, ${ }^{6,7}$ and potent inducers of apoptosis, including sulfasalazine, steroids, and azathioprine are efficacious therapies in patients with CD. ${ }^{8,9}$ The most established biological agents that induce apoptosis in lamina propria mononuclear cells (LPMC) in CD are the anti-TNF- $\alpha$ mAb infliximab and adalimumab. Randomized controlled trials have proven efficacy of this treatment in CD patients, whereas recent data suggest that apoptosis induction in the intestinal mucosa might be even predictive of therapeutic success. ${ }^{10,11}$

CD44 is a cell surface glycoprotein involved in multiple cellular functions, including cell-matrix interactions, cell migration, programmed cell death, and cellular activation. CD44 and its many variant isoforms were shown to exert some of their functions through docking and presentation of cytokines or growth factors to their cognate cell surface receptors or substrates. ${ }^{12}$ In contrast to the CD44 standard isoform, devoid of any variant regions, which is expressed ubiquitously in the healthy gut, we have shown that CD44v7 expression is mainly restricted to activated leukocytes in the inflamed mucosa. ${ }^{13}$ The region encoded by exon $v 7$ appears pivotal in experimental colitis, because treatment with antiCD44v7 antibodies led to complete remission in trinitrobenzene sulfonic acid (TNBS)- or dextrane sulfate sodium (DSS)induced colitis, in contrast to a CD44v6- or CD44v4-specific therapy. ${ }^{14,15}$ Also mice genetically deleted of CD44v7, by retaining complete expression of the remaining CD44 exons, were protected from TNBS-induced colitis. ${ }^{7}$ Furthermore, no colitis was observed when CD44v7-deficient mice were crossed with IL-10-deleted mice that spontaneously develop colitis. In all these models of experimental colitis, mice with CD44v7 targeting or deletion showed increased apoptosis in the inflamed mucosa. ${ }^{7}$ This is compatible with a function of the CD44v7 isoform in the suppression of apoptosis in inflammatory diseases. ${ }^{7,16}$

Since apoptosis induction of effector T cells is supposed to restore homeostasis in the intestinal mucosa, we investigated the regulation of CD44v7 expression in LPMC of patients with

\footnotetext{
${ }^{1}$ Medical Clinic 1, Department for Gastroenterology, Infectiology and Rheumatology, Charité University Medicine Berlin, Campus Benjamin Franklin, Berlin D 12200, Germany; ${ }^{2}$ Department for Gastroenterology, Hepatology, Nutrition, Marienhospital Altenessen, Essen D 45329, Germany and ${ }^{3}$ Department of Clinical and Biological Sciences, Institute for Medical Microbiology, University of Basel, Basel CH 4003, Switzerland

*Corresponding author: BM Wittig, Medical Clinic I, Campus Benjamin Franklin, Charité University Medicine Berlin, Hindenburgdamm 30, Berlin D-12200, Germany. Tel: + 49308445 4316; Fax: + 49308445 4481; E-mail: bianca.wittig @ charite.de

Keywords: CD44v7; inflammatory bowel disease; inflammation; macrophage differentiation; apoptosis; lamina propria

Abbreviations: CD, Crohn's disease; IBD, inflammatory bowel disease; LPMC, lamina propria mononuclear cells; DSS, dextrane sulfate sodium; UC, ulcerative colitis Received 04.9.06; revised 14.3.07; accepted 15.3.07; Edited by G Rabinovich; published online 04.5.07
} 
inflammatory bowel disease (IBD). We demonstrated that outside-in signals through CD44v7 elicited by anti-CD44v7 $\mathrm{mAb}$ resulted in increased apoptosis of human LPMC from CD patients and inhibition of macrophage activation. Moreover, we identified molecules involved in these signaling events and anticipate therapeutic use of anti-CD44v7 mAbs as a specific and efficient treatment strategy for CD patients.

\section{Results}

CD44 expression in inflamed mucosa from CD patients. In this study, we first have investigated the expression of CD44V isoforms in LPMC from normal intestinal mucosa and in inflamed mucosa of $C D$ and ulcerative colitis (UC) patients. LPMC from healthy controls showed little expression of the CD44 variant isoforms CD44v4 (median 9\%, range 3-11\%), CD44v6 (median 8\%, range $2-10 \%$ ) and $\mathrm{CD} 44 \mathrm{~V} 7$ (median 10\%, range 6-15\%) (Table 1). Corroborating our data in experimental colitis, ${ }^{5}$ in CD there is a slight upregulation of CD44v6 (median 15\%, range $2-24 \%$, while CD44v7 isoforms are significantly upregulated (median 27\%, range 16-35\%) (Table 1, Figure 1a). CD44v4 is only slightly increased (median 16\%, range 10-17\%) (Table 1). Interestingly, the panCD44 antibody revealed decreased frequency of total CD44 expression in CD patients (median $72 \%$, range $56-82 \%$ ) as compared to normal mucosa (median 91\%, range 75-97\%) and UC (median 88\%, range 78-96\%). This difference was significant $(P<0.05)$, and indicated an even more pronounced relative increase of CD44v7-positive cells (Figure 1a). CD44v expression was not significantly upregulated in LPMC of UC patients, as compared to mucosa from normal and CD patients (Table 1, Figure $1 \mathrm{~b}$ and $c)$. In LPMC from inflamed mucosa of CD patients, CD44v7 was expressed in CD4-positive T cells as well as in CD33-positive intestinal macrophages (data not shown). In a subset of patients (six patients with CD, four with UC and six controls) expression of the variant isoforms CD44v3 and CD44v10 was examined in LPMC by FACS. Both variant isoforms were not detected on freshly harvested LPMC ( $<1 \%$ positive cells) of patients and controls. We conclude that expression of $\mathrm{CD} 44 \mathrm{v} 7$ isoforms was significantly increased in inflamed mucosa from CD patients.

Ligation of CD44v7 induces apoptosis of LPMC from inflamed mucosa in CD, but not in UC or normal mucosa. Binding of a CD44v7-specific mAb-induced apoptosis in LPMC from inflamed mucosa (median relative apoptosis induction 1.61 (arbitrary units - incubation with an unspecific isotype control IgG was set to 1), range 1.2-2.6), as compared to non-inflamed mucosa of CD patients (median 0.97, range $0.8-1.5)(P<0.05)$ or control tissue (median 1.15, range 0.6-2.4) $(P<0.01$; Table 2). Apoptosis induction seemed to be closely associated with the presence of CD44V7 since this effect was not detected in LPMC of patients with active UC (Figure 2). The apoptotic effect was less pronounced following incubation with anti-panCD44 antibodies (clone IM7), but not detected with anti-CD44v4 or anti-CD44v6 antibodies (Table 2). An apoptosis-inducing anti-Fas antibody revealed cell death in all cell populations analyzed, which reached statistical significance in LPMC from non-inflamed CD mucosa, as compared to normal mucosa (Table 2). Only ligation of CD44v7 specifically induced cell death in inflamed mucosa of CD patients. Hence, CD44v7 is likely to play a major role in the immune pathogenesis of $C D$, comparable to our findings in experimental colitis in mice. ${ }^{7}$

Apoptosis can only be induced in pre-activated mononuclear cells. The apoptotic effect of the antiCD44v7-specific antibody in intestinal cells from inflamed mucosa but not in non-inflamed mucosa suggests that this $\mathrm{mAb}$ induces apoptosis predominantly in pre-activated CD44v7-positive immune cells. Consequently, CD44v7 expression was not detected in freshly prepared peripheral blood lymphocyte (PBL) (mean 1\% positive cells, range 0 $2 \%$, but slightly upregulated in CD3/CD28 pre-activated mononuclear cells of healthy controls (mean $1.5 \%$, range $0.8-1.7 \%$ ) and CD patients (mean $2.8 \%$, range $1.6-3.7 \%$ ) (data not shown). The anti-CD44v7 mAb was added to the pre-activated cells after 3 days for another $24 \mathrm{~h}$. In these cells with increased CD44v7 expression, apoptosis was induced in lymphocytes (median 1.4, range 1.0-2.4; Figure 3a). We also show that apoptosis induction was not accompanied by a significant inhibition of proliferation (median 10\% inhibition, range $0-14 \%$, data not shown). Apoptosis induction in PBL was dose dependent and increased with growing concentrations of anti-CD44v7 mAb from 1.1 to 1.4. At a concentration of $10 \mu \mathrm{g} / \mathrm{ml}$ anti-CD44v7 mAb, this difference reached statistical significance $(P<0.05$; Figure $3 \mathrm{~b})$.

Since LPMC of inflamed mucosa have a memory phenotype, we were interested to evaluate the susceptibility of memory cells in general to apoptosis as compared to naïve cells. To obtain memory and naive T cells in sufficient amount and viability PBLs were sorted accordingly and activated via anti-CD3 and anti-CD28. After the activation period, $10 \mu \mathrm{g} / \mathrm{ml}$

Table 1 Increased expression of CD44v7 in LPMC of inflamed mucosa in patients with CD

\begin{tabular}{|c|c|c|c|c|c|}
\hline \multirow[b]{2}{*}{ Mucosa } & \multirow[b]{2}{*}{$n$} & \multicolumn{4}{|c|}{$\%$ positive cells } \\
\hline & & panCD44 & CD44v4 & CD44v6 & CD44v7 \\
\hline $\begin{array}{l}\text { CD } \\
\text { UC } \\
\text { Normal }\end{array}$ & $\begin{array}{l}22 \\
13 \\
14\end{array}$ & $\begin{array}{l}72(56-82) \\
88(78-96) \\
91(75-97)\end{array}$ & $\begin{array}{c}16(10-18) \\
10(4-12) \\
9(3-11)\end{array}$ & $\begin{array}{r}15(2-24)^{\star} \\
12(6-18) \\
8(2-10)\end{array}$ & $\begin{array}{l}27(16-35)^{\star} \\
15(9-21) \\
10(6-15)\end{array}$ \\
\hline
\end{tabular}

Abbreviations: CD, Crohn's disease; UC, ulcerative colitis Flow cytometric analysis. Median (range) of CD44-positive cells is given normal: samples obtained from 14 colorectal cancer patients. Significance CD/normal mucosa, ${ }^{*} P<0.05$ 
a Crohn's disease
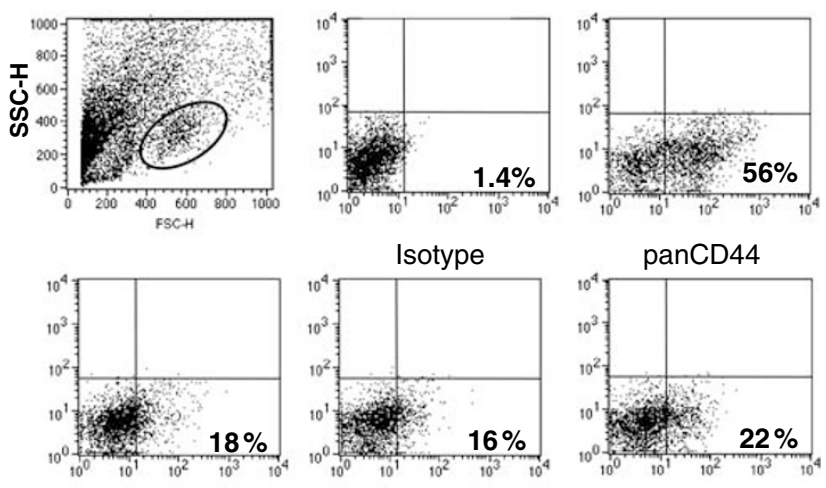

CD44v4

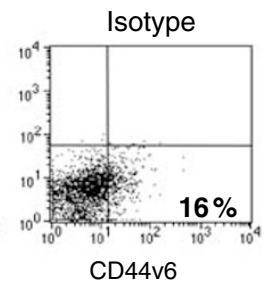

panCD44

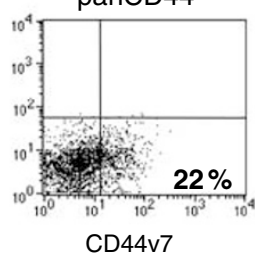

b Ulcerative colitis
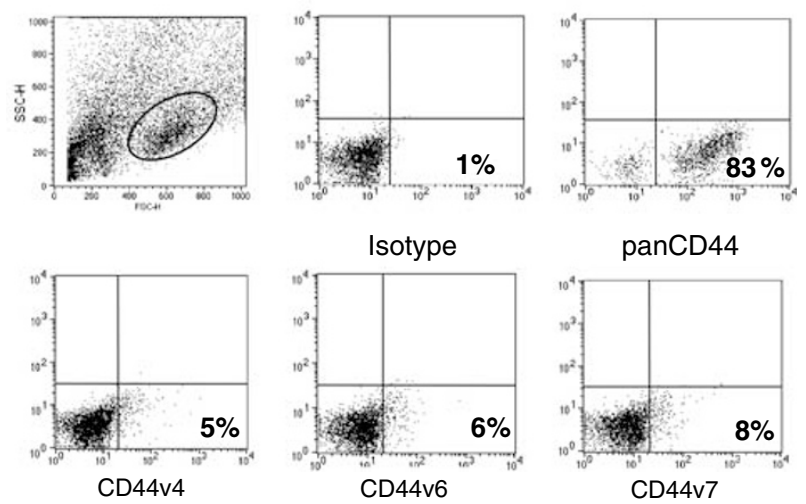

panCD44

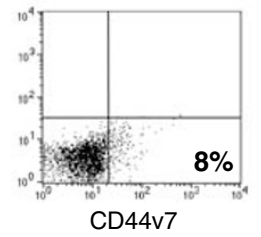

C Normal control
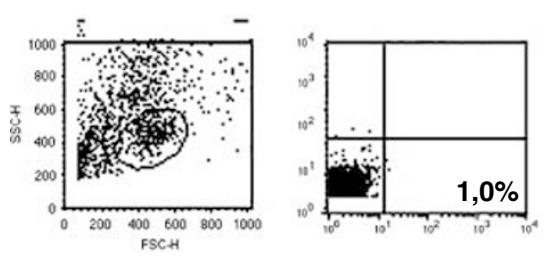

Isotype

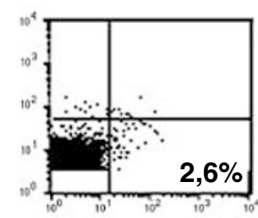

CD44v4

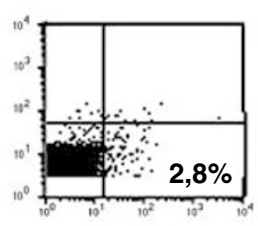

CD44v6

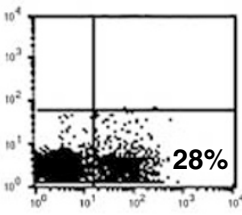

panCD44

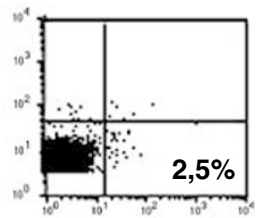

CD44v7
Figure 1 LPMC from CD patients express high levels of CD44v isoforms in contrast to UC patients. FACS analysis was performed on freshly isolated LPMC from biopsies of patients with active $C D$, active UC, and colorectal cancer. Lymphocytes and macrophages were gated and stained for total CD44 (panCD44), CD44v4, CD44v6, and CD44v7 expression. Mouse lgG1 served as isotype control. Representative data are shown. (a) CD for 4 years, active disease, treatment with Mesalamine. (b) UC, active disease, before treatment (c) Normal control refers to colorectal cancer patients

anti-CD44v7 antibody was added and incubated for another $24 \mathrm{~h}$. Indeed, CD45RO memory cells revealed increased susceptibility to apoptosis induction (mean 1.5, range 1.41.5 ), whereas naïve cells were resistant (mean 0.9 , range
0.9-1.0) (Figure 3c shows a representative FACS analysis). This indicates a specific effect of anti-CD44v7 antibody treatment to memory cells, which are the major T-cell population in IBD. ${ }^{17}$ Thus we demonstrate that in CD patients, these infiltrates consist of chronically activated CD44v7positive cells in a Th1 dominated immune response. Only pre-activated, CD44v7-positive cells can be induced by antiCD44v7 antibody to undergo cell death.

Ligation of CD44v7 prevents CD40-mediated activation of antigen-presenting cells. As we have shown before, CD40 ligation leads to rapid upregulation of CD44v7 in murine LPMCs. ${ }^{7}$ Here we show that CD40 ligation strongly induced CD44v7 expression in human macrophages from CD patients as well (Figure 4a). As a consequence, upon CD40 ligation, increased CD44v7 expression rendered monocytes more susceptible to anti-CD44v7-induced apoptosis (unstimulated cells were set to 1; CD40 ligation mean 0.7, range 0.6-1.0; CD40 ligation plus anti-CD44v7 $\mathrm{mAb}$ median 1.9, range 1.7-2.4) (Figure $4 \mathrm{~b}$ shows one representative evaluation). In dendritic cells, it is known that the costimulatory molecules CD80 and CD86 are upregulated upon CD40 ligation. ${ }^{18}$ Further, we show for the first time in human monocyte-derived dendritic cells (see Materials and Methods) that CD40 ligand-induced upregulation of CD80 expression was prevented by simultaneous addition of anti-CD44v7 mAb in vitro (Figure 4c). Dendritic cells from CD patients have a mean of $69 \%$ CD80-positive cells (range 62-78); dendritic cells treated with anti-CD $44 \mathrm{v} 7$ show a mean of $67 \%$ positive cells (range 62-73); dendritic cells with CD40 ligation show a mean of $79 \%$ positivity (range: $73-83$ ); while dendritic cells treated with CD40L and anti-CD44v7 mAb revealed a significantly reduced expression of $53 \%$ CD80-positive cells (range 49-58). This effect was detected before and independent of apoptosis induction. Thus, co-ligation of CD44v7 and CD40 diminished the maturation of antigenpresenting cells reflected by reduced CD80 expression.

CD44v7 regulates the mitochondrial pathway of apoptosis induction. The intrinsic apoptosis pathway is under close control of $\mathrm{Bcl}$ molecules. In fact, in LPMC of inflamed mucosa from CD patients, incubation with antiCD44v7 antibody was associated with a significant downregulation of anti-apoptotic Bcl-2 and an increase, however not significant, of the proapoptotic protein Bax (Figure 5a). Accordingly, anti-CD44v7 treatment induced the mitochondrial protein $7 A 6$ in inflamed mucosa of $C D$, which is detectable by the APO2.7 antibody at early stages of mitochondrial apoptosis. The relative upregulation of $7 A 6$ by anti-CD44v7 mAb was 1.51 (range 1.3-1.7) as compared to the isotype control (set to 1.0). One representative experiment is shown in Figure $5 \mathrm{~b}$. We next assessed activation of caspases in LPMC. As shown in Figure $5 \mathrm{c}$, incubation with anti-CD44v7 $\mathrm{mAb}$ for $24 \mathrm{~h}$ led to an increase of active caspase-3 compared to control IgG (median 1.8, range 1.13-2.5). Furthermore, the broad-spectrum caspase inhibitor Z-VAD-FMK suppressed anti-CD44v7 mAb-induced apoptosis completely (Figure $5 \mathrm{~d}$ ). A specific inhibitor for caspase-9, Z-LEHD-FMK, supported the findings from above 
Table 2 Apoptosis induction in LPMC from patients with CD

In vitro treatment

\begin{tabular}{|c|c|c|c|c|c|c|c|}
\hline \multirow[b]{2}{*}{ Mucosa } & \\
\hline & Control IgM & Anti-Fas & Control IgG & Anti-CD44v4 & Anti-CD44v6 & Anti- CD44v7 & Anti- panCD44 \\
\hline $\begin{array}{l}\text { CD Inflamed } \\
\text { CD non-inflamed } \\
\text { Normal mucosa }\end{array}$ & $\begin{array}{l}1.00 \\
1.00 \\
1.00\end{array}$ & $\begin{array}{r}1.50(1.2-2.1) \\
1.83^{*}(1.3-4.0) \\
1.45(0.9-2.3)\end{array}$ & $\begin{array}{l}1.00 \\
1.00 \\
1.00\end{array}$ & $\begin{array}{l}1.18(1.0-1.3) \\
1.09(1.0-1.1) \\
1.11(1.0-1.2)\end{array}$ & $\begin{array}{l}1.15(0.9-1.4) \\
1.04(0.8-1.3) \\
1.05(0.9-1.2)\end{array}$ & $\begin{array}{r}1.61^{\star \star}(1.2-2.6) \\
0.97(0.8-1.5) \\
1.15(0.6-2.4)\end{array}$ & $\begin{array}{c}1.31^{*}(1.0-2.2) \\
1.14(0.9-2.3) \\
1.17(1.0-2.2)\end{array}$ \\
\hline
\end{tabular}

$\mathrm{CD}$, Crohn's disease Median (range) of 14 experiments. Apoptosis induction is given as the relative rate of spontaneous apoptosis in cells treated with control IgG or $\operatorname{lgM}(=1)$ after incubation for $20 \mathrm{~h}$. Evaluation of apoptosis was determined as described by Nicoletti et al ${ }^{37}$ Significance of inflamed/non-inflamed mucosa and noninflamed/control, resp.: * $P<0.05$, ${ }^{\star *} P<0.01$

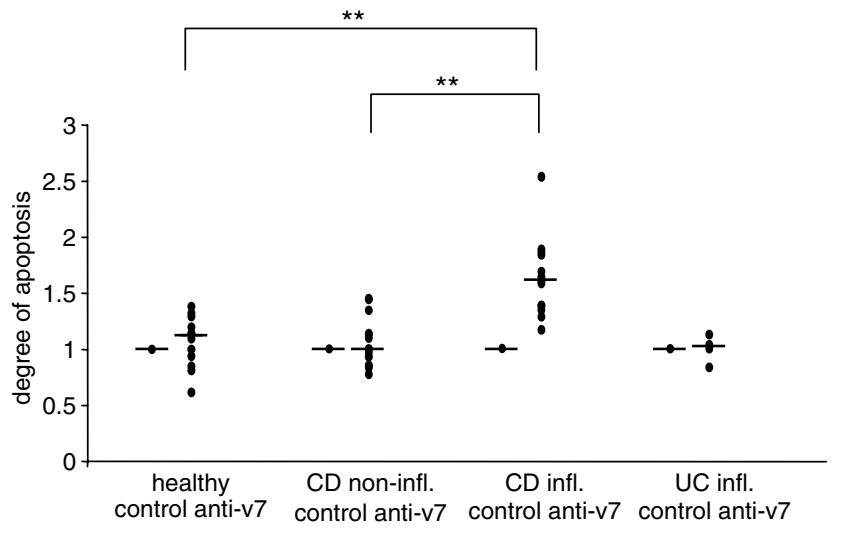

Figure 2 Apoptosis can be specifically induced in LPMC from patients with CD. Apoptosis of LPMC from control tissue $(n=14)$, non-inflamed and inflamed mucosa from CD patients $(n=14)$ as well as inflamed tissue from UC patients $(n=4)$ after treatment with control IgG mAb or after incubation with $10 \mu \mathrm{g} / \mathrm{ml}$ anti-CD44v7 mAb for $24 \mathrm{~h}$ was evaluated. Apoptosis was evaluated by PI staining in hypotonic solution and analyzed by flow cytometry. ${ }^{37}$ Arbitrary units are given (incubation with an unspecific isotype control IgG was set to 1). Individual results are shown as points, lines display the mean apoptosis induction in comparison to control. Significance ${ }^{* *} P<0.01$. Control: control IgG; $\alpha$ CD44v7 : anti-CD44v7 antibody

that anti-CD44v7 mAb-induced cell death occurred via the mitochondrial apoptosis pathway. The apoptosis induction in LPMC was blocked to $80 \%$ by the caspase- 9 inhibitor (Figure 5d). Furthermore, anti-CD44v7 treatment upregulated the expression of the caspase-9 adaptor protein APAF-1 in activated LPMC from patients with CD (huLPMC), in activated PBL (huPBL), as well as in activated spleen cells from mice (muSpl) in comparison to untreated cells (Figure 5e). Hence, apoptosis induction through CD44v7 signaling is mediated via the mitochondrial pathway.

The CD44v7 and Fas-apoptotic pathways are independent. It has been described before that Fas ligation does not induce apoptosis in inflamed mucosa of CD patients. $^{9}$ In line with this, we observed that Fasmediated apoptosis was markedly lower in LPMC from inflamed as compared to LPMC from non-inflamed mucosa in CD patients (Table 2). However, we were interested to find out whether CD44v7-induced apoptosis might be dependent on Fas signaling. We found that pre-incubation of LPMC with a Fas neutralizing antibody (clone ZB4) specifically blocked Fas-mediated apoptosis but not the anti-CD44v7 effect (Figure 6a). Similarly, co-incubation of LPMC with both antibodies, Fas and against CD44v7, revealed an additive apoptosis-inducing effect (data not shown). In a further study, we used mononuclear spleen cells from 6-week-old female $\mathrm{MRL} / \mathrm{lpr}$ mice, deficient for Fas receptor, BALB/c mice deficient for CD44v7, and BALB/c control mice, stimulated them for 3 days via anti-CD3 and anti-CD28, followed by treatment with the mouse-specific anti-CD44v7 antibody LN7.1 $(20 \mu \mathrm{g} / \mathrm{ml})$ or a corresponding IgG control for $24 \mathrm{~h}$. Our data indicate that Fas ligation is not required for antiCD44v7-induced apoptosis, because anti-CD44v7 treatment induced cell death in spleen cells from Fas-deficient mice (Figure 6b).

\section{Discussion}

In the current study, we demonstrated that antibody targeting/ ligation of CD44v7 in vitro led to the induction of apoptosis. This effect was specific for activated, but not for resting lamina propria $T$ cells and macrophages of patients with active CD. $\mathrm{CD}$ is known as a Th1-mediated chronic inflammation. ${ }^{19}$ Our data suggest that targeting CD44v7 may constitute a novel and effective approach to induce apoptosis in activated mononuclear cells in vivo. Such an approach may be useful in the treatment of autoimmune diseases, and chronic inflammation in general.

Importantly, treatment with the anti-CD44v7 antibody did not induce apoptosis of lamina propria $T$ lymphocytes or macrophages from either healthy individuals, non-inflamed mucosa from CD or in the inflamed mucosa of UC patients because these cells did not express significant amounts of CD44v7. The ability to induce apoptosis was highly specific for CD44v7-positive cells, as opposed to antibodies against CD44v4 or CD44v6, both variants being also upregulated in inflamed mucosa in $C D$. These findings may have implications for the design of novel specific therapies for IBD, since direct binding of the anti-mouse ${ }^{16}$ and anti-human CD44v7 antibodies revealed similar biologic effects in both species and likewise resulted in apoptosis induction.

Several reports have shown that signaling through CD44 can protect from apoptosis in various tumor cells ${ }^{20,21}$ but there are also reports that CD44 ligation leads to enhanced apoptosis. ${ }^{22}$ These apparent discrepancies may be due to the fact that the antibodies have different functional activities (blocking or activating) and that CD44 exists in multiple variant isoforms that are upregulated under highly restricted conditions during inflammation and tumorigenesis ${ }^{23,24}$ where they provide a pro-survival capacity to the cells. ${ }^{7}$ In most of the reports, the isoforms were not distinguished. The only known ligand for the region CD44v6/v7 is osteopontin, a secreted 
a Apoptosis of activated PBL

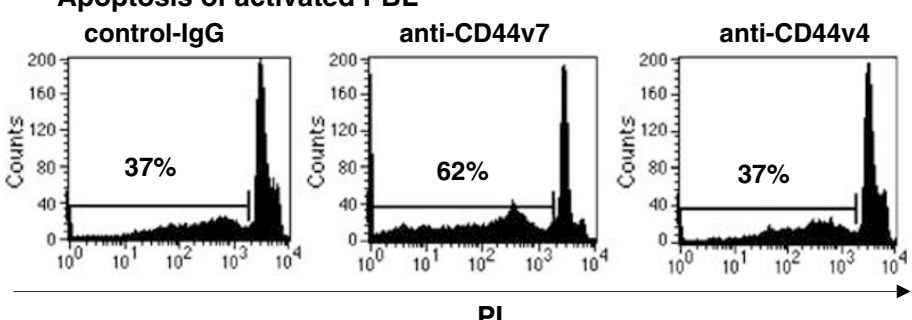

PI

b Dose dependent apoptosis induction

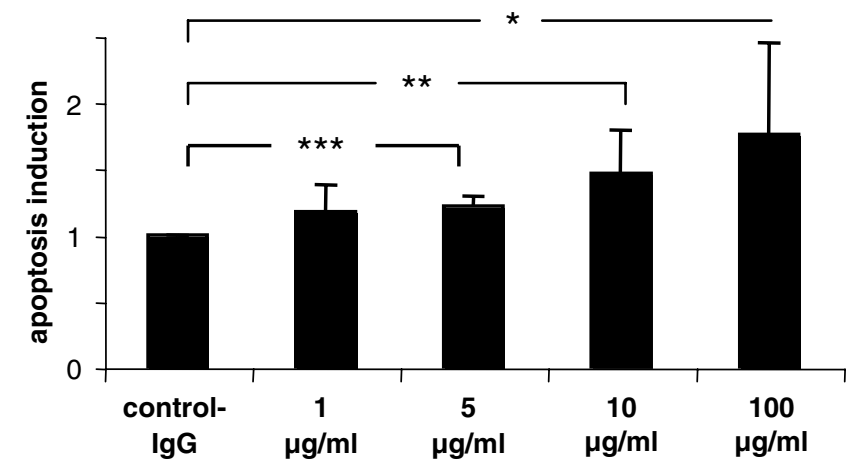

C Induction of apoptosis in naive or memory cells Naive cells

Memory cells
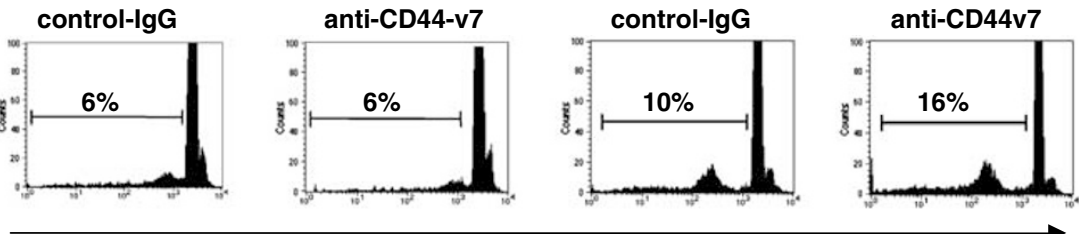

Figure 3 Induction of apoptosis in activated PBL and memory T cells. PBL of healthy donors were activated by anti-CD3 and anti-CD28 treatment. On day 3 of activation, $10 \mu \mathrm{g} / \mathrm{ml}$ of either anti-CD44v7 mAb, anti-CD44v4 mAb, or isotype control was added to the cells for a further incubation period of $24 \mathrm{~h}$. (a) Apoptosis was evaluated by PI staining in hypotonic solution and analyzed by flow cytometry as described by Nicoletti et al. ${ }^{37}$ Shown are FACS data from one out of nine experiments. (b) Dose-dependent rate of apoptosis induction by $\mathrm{CD} 44 \mathrm{v} 7 \mathrm{mAb}(1,5,10$, and $100 \mu \mathrm{g} / \mathrm{ml})$. The isotype control (control-lgG) was set to 1. Bars: median of 3-9 experiments. Significance ${ }^{*} P<0.05$. (c) Induction of apoptosis in activated naive or memory T cells. Cells were sorted as described in Materials and Methods, activated by anti-CD3 and anti-CD28 and treated for $24 \mathrm{~h}$ with $10 \mu \mathrm{g} / \mathrm{ml}$ anti-CD44v7 mAb or isotype control. Apoptosis was evaluated by PI staining in hypotonic solution and analyzed by flow cytometry as described by Nicoletti et $a l^{37}$ Shown are FACS data from one out of three experiments

phosphoprotein involved in Th1 responses of chronic and autoimmune inflammatory diseases. ${ }^{25}$

What is the evidence for a specific CD44v7 involvement in the inflamed mucosa in CD patients? Apoptotic signaling generally proceeds through the extrinsic and intrinsic pathways. Initiation of either pathway leads to the activation of a cascade of proteolytic caspases that mediate cell destruction. ${ }^{26}$ In our study in CD patients, one of the early signs indicating apoptosis induction denotes disruption of the mitochondrial membrane. This process was evident by upregulated expression of the mitochondrial membrane protein $7 A 6$ in anti-CD44v7 mAb-treated LPMC and peripheral blood mononuclear cells (PBMC). Moreover, mitochondrial homeostasis requires a balance between proapoptotic and anti-apoptotic members of the Bcl-2 family. ${ }^{27}$ Remarkably, this balance was regulated towards proapoptosis in anti-CD44v7-treated LPMC by a downregulation of Bcl2. The disturbed mitochondrial integrity induced activation of
APAF-1 that subsequently activated the cell death pathways via caspases- 9 and -3 . In patients with $C D$, but not UC, enhanced expression of anti-apoptotic $\mathrm{Bcl}$ molecules has previously been described. ${ }^{5}$ Therefore, we suggest that signaling through CD44v7 interferes with expression of antiapoptotic molecules of the $\mathrm{Bcl}$ family, which explains the proapoptotic effect in LPMC from CD patients, in contrast to UC patients.

Another explanation that CD44v7 is predominantly upregulated in $C D$ but not UC relates to the cytokine response in these diseases. CD inflammation is driven by a Th1 immune response, whereas $\mathrm{T}$ cells in UC produce Th2 cytokines. $^{19}$ Further, in CD, an IL-17-producing T-cell population, likely driven by IL-23, has been identified that significantly contributes to tissue damage in the intestine. ${ }^{28}$ In experimental colitis, we have shown that CD44v7 is essential in various Th1-mediated models. ${ }^{7,14,15}$ Furthermore, in patients with progressive alopecia areata, another Th1-mediated 

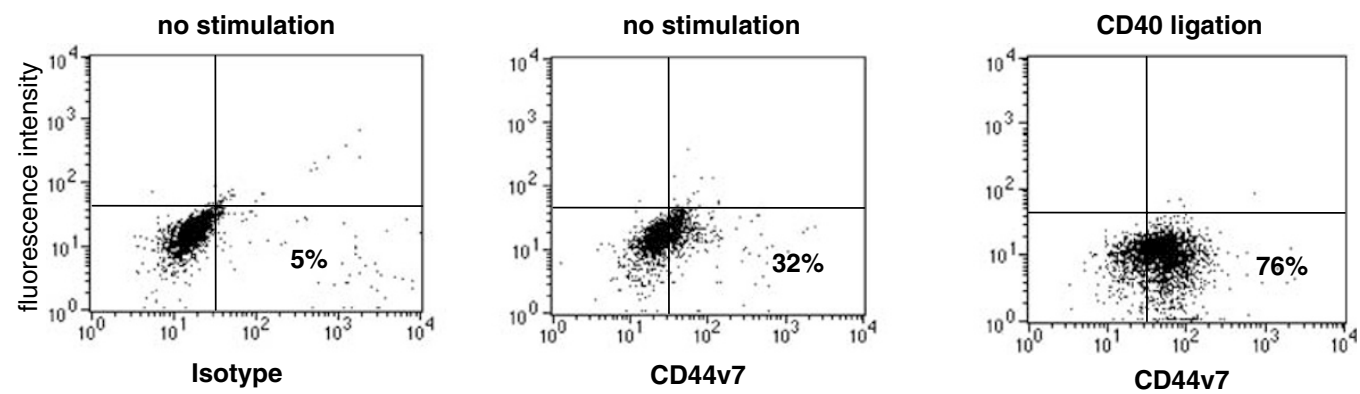

b

Anti-CD44v7 antibody induces apoptosis in macrophages activated via CD40 ligation
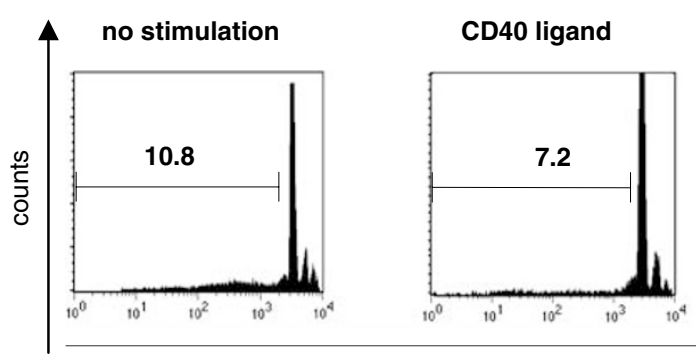

CD40 ligand

+anti-CD44v7

PI

C

Down-regulation of CD80 by anti-CD44v7 in mature dendritic cells

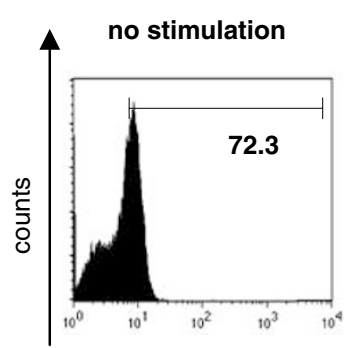

anti-CD44v7

CD40 ligand

CD40 ligand+
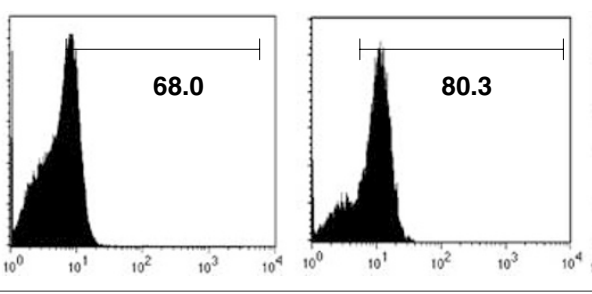

anti-CD44v7

CD80

Figure 4 Effects of CD40 ligation on macrophages and monocyte-derived dendritic cells. Monocytes or monocyte derived dendritic cells from PBLs were incubated for $24 \mathrm{~h}$ with soluble CD40 ligand $(5 \mu \mathrm{g} / \mathrm{ml})$ and anti-CD $44 \mathrm{v} 7 \mathrm{mAb}$ where indicated $(10 \mu \mathrm{g} / \mathrm{ml})$. Shown are representative data from five independent experiments. (a) Ligation of $\mathrm{CD} 40$ with a soluble CD40L fusion protein induces CD44v7 expression in macrophages. (b) CD44v7 blockade induces apoptosis in macrophages activated via CD40 ligation. Apoptosis was determined by PI staining in hypotonic solution. Shown are representative data from five independent experiments. (c) CD40 ligation enhances dendritic cell maturation, indicated by upregulation of $\mathrm{CD} 80$ that is abrogated by anti-CD44v7 mAb. Shown are representative data from four independent experiments

autoimmune disease, inflammatory infiltrates are composed mainly of CD25/CD154-positive CD4 T cells that express CD44V7 and display increased resistance towards apoptosis. $^{29}$ In the mouse model of alopecia areata, ${ }^{30}$ apoptosis resistance was accompanied by $\mathrm{Bcl}-2$ and $\mathrm{Bcl}-\mathrm{XL}$ upregulation. Although not analyzed, it is tempting to speculate that anti-CD44v7 antibody might induce apoptosis in these infiltrates too.

We have described earlier that in LPMC from CD patients and mice with colitis, CD44v7 functions as a costimulatory molecule that is upregulated upon ligation of the costimulatory molecule CD40. ${ }^{7,31}$ In patients with CD, but not UC, dendritic cells from inflamed tissue express significantly higher levels of maturation and activation markers CD80/CD86 and CD40. ${ }^{32}$ The apoptosis-preventing effect of CD40 ligation has been related to upregulation of the anti-apoptotic proteins $\mathrm{Bcl}-2$ and $\mathrm{Bcl}-\mathrm{XL}$, which counter-balance the apoptotic property of various maturation stimuli in dendritic cells. ${ }^{33}$ Moreover, blocking of CD40/CD154 ligation is an effective means to ameliorate autoimmune diseases. ${ }^{34}$ In accordance with our studies in experimental colitis, ${ }^{7}$ we could show here that in monocytes from CD patients, CD44v7 expression was upregulated following CD40 ligation with CD154 (CD40L). As a consequence, the apoptosis-inducing ability of the CD44v7 antibody was more evident in CD40-activated mononuclear cells. Furthermore, before apoptosis induction, anti-CD44v7 antibody application at the time of CD40 ligation induced downregulation of the disease-related increase of the activation marker CD80 on dendritic cells in CD patients.

Thus, the apoptosis-inducing effect in both models, deletion of CD44v7 in the mouse or antibody targeting in LPMC cells from CD patients might be the result of abrogating the CD44v7 interaction with the CD40 receptor-ligand system, the latter being responsible for survival of activated cells in chronic 
a $\mathrm{Bcl}-2$ and $\mathrm{Bax}$ expression

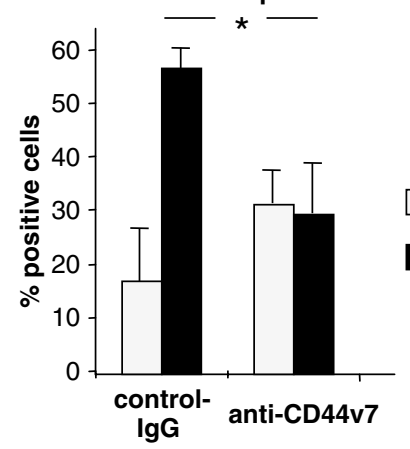

b

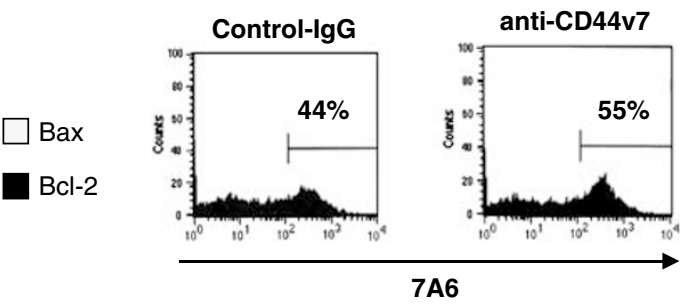

C

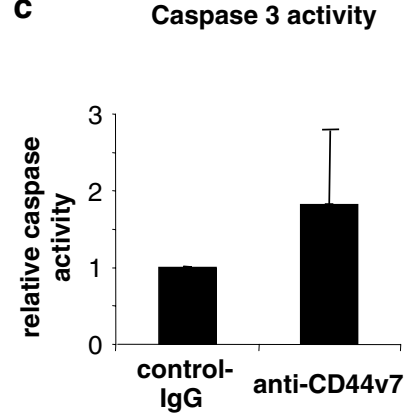

Caspase 3 activity

d Caspase inhibitors

e Expression of APAF-1
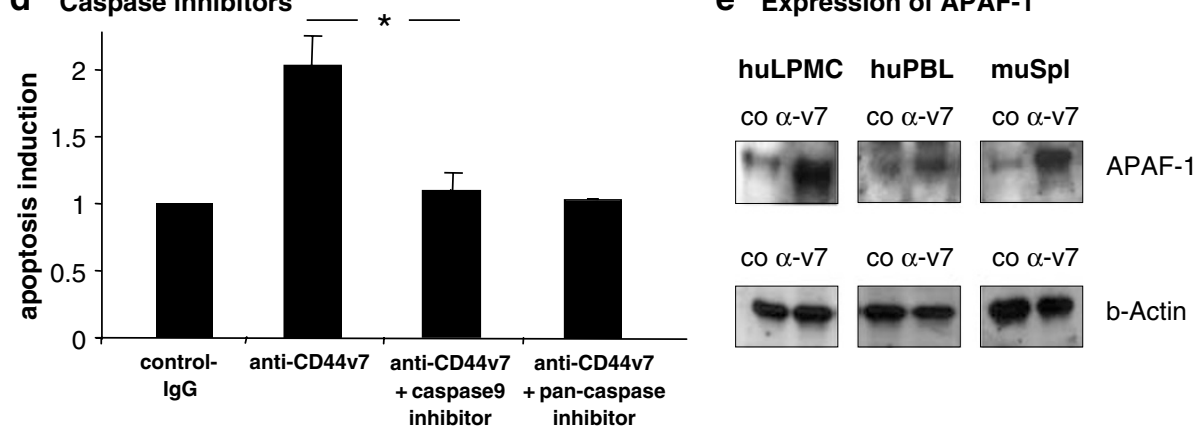

Figure 5 Anti-CD44v7 induces mitochondrial apoptosis in LPMC. (a) Flow cytometric detection of Bax/Bcl-2 in LPMC from inflamed mucosa of CD patients. Cells were incubated with control lgG (negative control) or anti-CD44v7 mAb (10 $\mu \mathrm{g} / \mathrm{ml})$ for $20 \mathrm{~h}$. Bax and Bcl-2 were detected intracellularly and analyzed by FACS. Bars: mean \pm S.D. from five CD patients. (b) For detection of the early apoptotic marker 7A6, antigen cells were fixed, permeabilized, and stained with anti-7A6 (clone Apo2.7). Shown are representative FACS data from one out of three experiments. (c) Caspase-3 activity was determined in LPMC of CD patients treated with anti-CD44v7 mAb (10 $\mu \mathrm{g} / \mathrm{ml})$ and control IgG. Bars: mean \pm S.D. from one out of three experiments. (d) Inhibiting apoptosis signaling by specific caspase inhibitors prevents anti-CD44v7 mAb-induced apoptosis. LPMC from patients with CD were pre-incubated for $1 \mathrm{~h}$ with $10 \mu \mathrm{M}$ specific caspase inhibitors: Z-LEHD-FMK (caspase-9 inhibitor) or broad-spectrum caspase inhibitor Z-VAD-FMK before adding control lgG or anti-CD44v7 mAb $(10 \mu \mathrm{g} / \mathrm{ml})(\alpha \mathrm{CD} 44 \mathrm{v} 7)$ for further $20 \mathrm{~h}$. Bars: mean \pm S.D. from five independent experiments. (e) Detection of APAF-1 by Western blotting. LPMC (huLPMC) and PBLs (huPBL) from patients with CD were treated with $10 \mu \mathrm{g} / \mathrm{ml}$ anti-human CD44v7 mAb and control lgG. Activated spleen cells from mice (muSpl) were incubated with $20 \mu \mathrm{g} / \mathrm{ml}$ anti-murine CD44v7 mAb (clone LN7.1) for $24 \mathrm{~h}$

inflammatory or autoimmune diseases. ${ }^{34}$ However, it is intriguing that modulating CD44v7 results in two different effects: absence of the molecule in the CD44v7-deleted mice might induce faulty activation and lack of migration of cells into the inflammatory sites in the colon, ${ }^{15,35}$ resulting in increased apoptosis. ${ }^{7,16}$ In contrast, the CD44V7 antibody might predominantly induce apoptosis signaling by withdrawal of the costimulatory signal CD44v7. Interestingly, only activated, but not naïve, cells were susceptible to anti-CD44v7 mAbinduced apoptosis. This supports our hypothesis that CD44v7 targeting by a specific antibody converts the costimulatory signal of CD44v7 into an apoptotic signal in CD patients.

Activation-induced cell death and Fas-FasL interaction have been shown to play a significant role in immune homeostasis. However, Fas-deficient mononuclear cells and cells from BALB/c mice were equally susceptible to apoptosis induced by the murine anti-CD44v7 mAb LN7.1. In addition, anti-CD44v7-mediated apoptosis was not abrogated by a Fas-blocking antibody, nor synergistically enhanced by a ligating Fas antibody. Although these results do not exclude the importance of Fas expression in apoptosis of lamina propria cells, they suggest that additional molecules, such as CD44v7, are independent of Fas to achieve cell survival in autoimmune disease with a Th1/Th17-driven immune response.

There is increasing evidence that induction of apoptosis in immune competent cells of the lamina propria has a beneficial effect in the treatment of CD. ${ }^{36}$ In the present work, we were able to prove a specific apoptosis-inducing effect by antiCD44v7 treatment in activated lamina propria macrophages and $\mathrm{T}$ lymphocytes from $\mathrm{CD}$ patients. In addition, we demonstrate that anti-CD44v7 mAb in vitro blocks the CD80 expression in monocyte-derived dendritic cells induced by CD40 ligation, which itself induces a potent Th1 polarization. ${ }^{18}$ Anti-CD44v7 treatment already proved to be beneficial in experimental colitis ${ }^{14,15}$ and the expression is highly restricted to inflamed gut of CD patients. Our results in human intestinal lamina propria cells provide a strong rationale for targeting CD44v7 in clinical trials.

\section{Materials and Methods}

Patients. We examined 35 patients with chronic IBD, 14 control patients with colorectal cancer and 4 patients with diverticulitis treated at the University Hospital of Saarland and the Charité - University Medicine Berlin, Campus Benjamin Franklin with approval of the institutional human research ethic committee following the Helsinki Guidelines. Twenty two patients (age 18-46) had CD and thirteen 
a

Fas independent apoptosis induction apoptosis induction

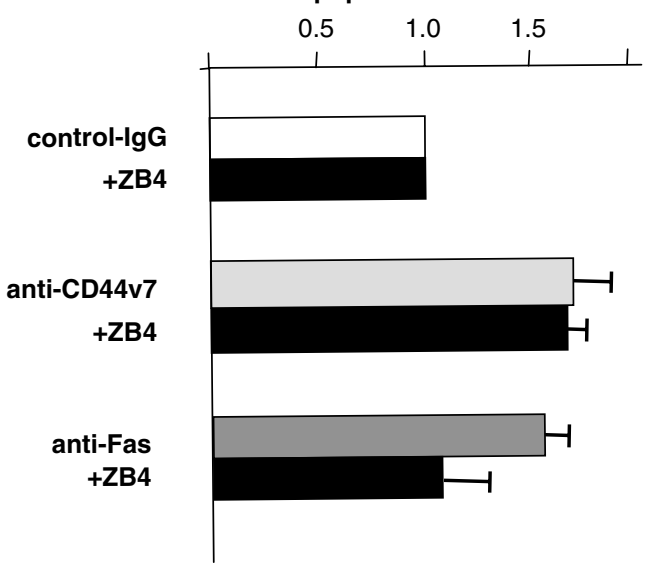

b Induction of apoptosis in mononuclear cells from BALB/c mice

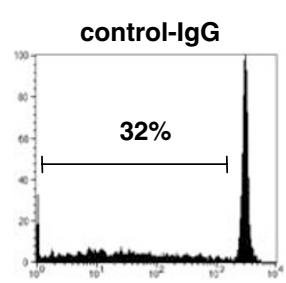

$20 \mu \mathrm{g} / \mathrm{ml} \mathrm{LN7.1}$

Fas deficient LPR mice

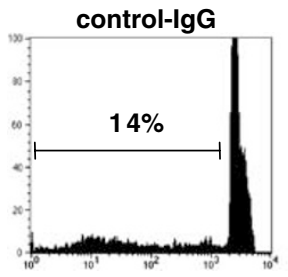

$20 \mu \mathrm{g} / \mathrm{ml} \mathrm{LN7.1}$

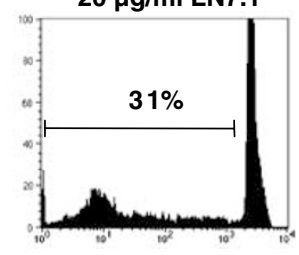

CD44v7 deficient mice

control-lgG

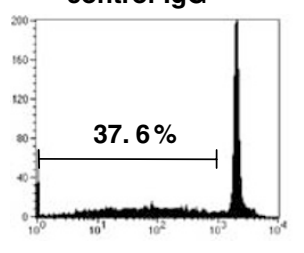

$20 \mu \mathrm{g} / \mathrm{ml}$ LN7.1

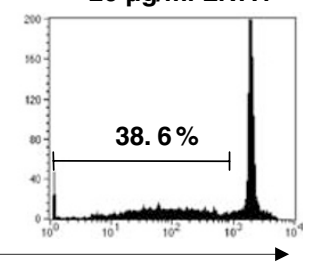

PI

Figure 6 Anti-CD44v7-induced apoptosis is independent of Fas ligation. (a) LPMC from patients with $C D$ were pre-incubated for $1 \mathrm{~h}$ with $100 \mathrm{ng} / \mathrm{ml}$ blocking antiFas $\mathrm{mAb}$ (clone ZB4) before adding control lgG, anti-CD44v7 mAb $(10 \mu \mathrm{g} / \mathrm{ml})$, or $100 \mathrm{ng} / \mathrm{ml}$ ligating anti-Fas mAb (clone $7 \mathrm{C} 11)$ for further $20 \mathrm{~h}$. Bars: mean \pm S.D. from three independent experiments. (b) Mononuclear cells from BALB/c wild-type and CD44v7 deficient mice and MLR/pr (Fas-deficient) mice were activated via anti$\mathrm{CD} 3$ and anti-CD28 and incubated for 1 day with mouse anti-mouse-CD44v7 mAb (clone LN7.1) or isotype control. CD44v7-deleted mice served as negative control. Shown are FACS data from one of three independent experiments

patients (age 29-52) had UC. Disease was active in 17/22 patients with CD and 10/ 13 patients with UC. CD patients were treated with aminosalicylates (20/22), with aminosalicylates and steroids (14/22), and patients received azathioprine and/or aminosalicylates and steroids (9/22) at the time of investigation. UC patients were treated with aminosalicylates (6/13), aminosalicylates and steroids (6/13), and/or azathioprine (4/13). A total of 14 out of 22 patients with CD underwent surgery and 5 out of 13 patients with UC were colectomized. Indications for surgery consisted of recurrent obstruction or stenosis in 10 patients, and fistulas or abscess formation in 4 patients in $\mathrm{CD}$; in $\mathrm{UC}$, intractable disease was the indication. All patients with colitis had an established diagnosis of CD or UC based on standard clinical, endoscopic, and histological criteria. Endoscopic biopsies or surgical specimen were taken from areas of active inflammation or normal appearing mucosa in the colon. Control specimens were taken from patients with colorectal cancer $(n=14$ patients, ranging from 38 to 77 years of age) at least $10 \mathrm{~cm}$ away from the tumor close to the resection margins and were macroscopically and microscopically normal. Four patients had sigma diverticulitis. Informed consent was obtained from all patients.

Isolation of lamina propria mononuclear and peripheral blood cells. LPMC from biopsies and surgically resected tissue were isolated as described before. In brief, intestinal biopsies and tissue were chopped into pieces and washed in PBS. This step was followed by an EDTA $(0.5 \mathrm{M})$ incubation period in a shaker at $4^{\circ} \mathrm{C}$ for $30 \mathrm{~min}$. Cells were washed and subsequently incubated in HBSS containing $1 \mathrm{mg} / \mathrm{ml}$ collagenase (Sigma-Aldrich), $200 \mu \mathrm{g} / \mathrm{ml}$ hyaluronidase (SigmaAldrich) and $100 \mu \mathrm{g} / \mathrm{ml}$ DNAse (Sigma-Aldrich) at room temperature for $10 \mathrm{~min}$. After washing, cells were separated through a needle and a $40 \mu \mathrm{m}$ cell strainer was used to remove cell debris. Where indicated, macrophages were enriched by plastic adhesion for $2 \mathrm{~h}$ at $37^{\circ} \mathrm{C}$. Nonadherent cells were removed by washing $3 \times$ with PBS, and adherent cells were cultured in RPMI 1640. LPMC viability was determined by Trypan blue staining. Human PBMC from healthy volunteers (28-41 years of age) were isolated using Ficoll-Hypaque gradients. In some cases, CD4positive $T$ cells from peripheral blood were enriched by staining with an FITClabeled anti-CD4 mAb (clone RPA-T4, BD Pharmingen, San Diego, CA, USA) followed by MACS using an anti-FITC MultiSort kit and LS-separation columns (both Miltenyi Biotech, Bergisch Gladbach, Germany). Subsequently, CD4-positive T cells were stained with PE-labeled anti-CD45RA mAb (clone HI100, BD Pharmingen) or anti-CD45RO mAb (clone UCHL1, BD Pharmingen) and sorted by anti-PE microbeads and LS-separation columns (Miltenyi Biotech). The purity was $>92 \%$ for CD4-positive CD45RA and >98\% for CD4-positive CD45RO population.

Expression of CD44 variant isoforms analyzed by flow cytometry. FACS analysis of mononuclear cells from peripheral blood and lamina propria was performed using directly PE-labeled antibodies against CD4 (DAKO, Hamburg, Germany), CD33 (Dianova, Hamburg, Germany) in LPMC, CD11b (Dianova), and FITC-labeled monoclonal antibodies against panCD44 (clone IM-7, rat anti-mouse, cross-reactive to human, IgG2b, ATCC, Manassas, VA, USA or clone SFF-2, BenderMedSystems, Vienna, Austria), and against variant isoforms CD44v3 (clone VFF-327v3), CD44v4 (clone VFF-11), CD44v6 (VFF18), CD44V7 (clone VFF9), and v10 (clone VFF-14) (all mouse-anti-human IgG1, BenderMedSystems). In all fluorescence studies, irrelevant monoclonal antibodies served as isotype controls. Cells were analyzed by four-color laser flow cytometry (FACS Calibur, Becton Dickinson, Heidelberg, Germany) using CellQuest ${ }^{\mathrm{TM}}$ software provided by Becton Dickinson. After forward-sideward scatter analysis, an electronic gate was added to include macrophages or T cells for analysis.

For determination of CD44 variant expression on naive or memory cells, PBL were double stained for $\mathrm{CD} 44$ variants with FITC-labeled $\mathrm{mAb}$ (see above) and PElabeled mAb against CD45RA (clone HI100, BD Pharmingen) or CD45RO (clone UCHL1, BD Pharmingen).

Cell stimulation. Isolated LPMC were resuspended in RPMI medium containing $10 \%$ fetal calf serum and cultured in 24-well plates at a concentration of $1 \times 10^{6} / \mathrm{ml}$. T-cell activation was provided by adding $1 \mu \mathrm{g} / \mathrm{ml} \mathrm{PHA}$ and recombinant IL-2 $(40 \mathrm{U} / \mathrm{ml})$, or $2 \mu \mathrm{g} / \mathrm{ml}$ plate-bound anti-CD3 monoclonal antibody (OKT3, ATCC, Manassas, VA, USA) for 3 days. PBL or purified CD4-positive cells were stimulated with $3 \mu \mathrm{g} / \mathrm{ml}$ plate-bound anti-CD3 monoclonal antibody (clone OKT3) and $1 \mu \mathrm{g} / \mathrm{ml}$ soluble anti-CD28 monoclonal antibody (clone CD28.2, BD Pharmingen) for 3 days. Macrophages were stimulated by $1 \mu \mathrm{g} / \mathrm{ml}$ LPS (SigmaAldrich) and $10 \mathrm{U}$ IFN- $\gamma$ (Chemicon International, Temecula, CA, USA) for 3 days.

Apoptosis induction and quantification of apoptotic cells. Apoptosis rate occurring spontaneously or after activation by various stimuli was examined. To determine induction of apoptosis in activated macrophages and T cells, a time kinetic (every $2 \mathrm{~h}$ from 4 to $24,48,72 \mathrm{~h}$ ) was 
performed and showed maximum apoptosis induction by anti-CD44v7 after $18-24 \mathrm{~h}$. Accordingly, freshly isolated LPMC were co-incubated for $20-24 \mathrm{~h}$ with the indicated amounts of antibody and then analyzed by FACS. For some experiments (Figure 3), in vitro-stimulated PBL were prepared by Ficoll separation and investigated regarding apoptosis evaluation. Antibodies used for apoptosis evaluation were a ligating monoclonal antibody against Fas $(100 \mathrm{ng} / \mathrm{ml}$ ) (clone 7C11, Coulter Immunotech, Krefeld, Germany), a blocking Fas antibody (100 ng/ml) (clone ZB4, Beckman Coulter), or antibodies against CD44 standard (clone IM-7 (ATCC) or clone SFF-2 (BenderMedSystems)), CD44v4 (VFF11), CD44v6 (VFF18), and CD44v7 (VFF9) (BenderMedSystems) $(10 \mu \mathrm{g} / \mathrm{ml}$ each). The percentage of apoptotic cells was determined by detection of hypodiploid cell nuclei after gating on DNA content as described by Nicoletti et al. ${ }^{37}$ After the incubation period, cells were resuspended in a fluorochrome hypotonic solution $(50 \mu \mathrm{g} / \mathrm{ml}$ propidium iodide $(\mathrm{PI})$ in $0.1 \%$ sodium citrate and $0.1 \%$ Triton $\mathrm{X}-100$ for at least $12 \mathrm{~h}$ at $4{ }^{\circ} \mathrm{C}$ in the dark. Finally, cells were analyzed on a FACSCalibur. Because of the fragmentation of DNA, apoptotic cells appear as hypodiploid in the DNA histogram. In our hands, the frequencies of apoptotic cells obtained in this assay are comparable to frequencies determined by the annexin $\mathrm{V}$ assay. Because the degree of apoptosis varied considerably between individual experiments (15-55\% apoptotic cells), most probably due to viability differences after the isolation procedure for LPMC, anti$\mathrm{CD} 44 \mathrm{v} 7 \mathrm{mAb}$-induced apoptosis was given as relative rate compared to control lgG.

\section{Activation of macrophages and dendritic cells by CD40 ligation. Monocytes were isolated from peripheral blood by CD14-positive sort (Miltenyi Biotec, Bergisch Gladbach, Germany). Dendritic cells were generated from isolated monocytes by culturing with granulocyte/macrophage colony stimulating factor $(10 \mathrm{ng} / \mathrm{ml})$ (Sigma, München, Germany) and IL-4 (100 ng/ml) (Chemicon, München, Germany). At day 5, a cocktail of pro-inflammatory cytokines and prostaglandins was added to induce maturation of the dendritic cells. ${ }^{38}$ For activation, recombinant soluble CD40L (BenderMedSystems) was added to the monocytes at day 1 and to the dendritic cells at day 5 for $24 \mathrm{~h}$. Where indicated, cells were incubated with anti-CD44v7 mAb, while anti-CD44v4 mAb served as isotype control $(10 \mu \mathrm{g} / \mathrm{ml}$ each). In mature dendritic cells, expression of the costimulatory molecule CD80 (Clone 2D10, eBiosciences, USA) was analyzed by FACS.}

\section{Intracellular staining of apoptosis-related mitochondrial proteins. For the intracellular detection of the mitochondrial proteins Bax and $\mathrm{Bcl}-2$, cells were fixed and permeabilized using a permeabilization kit (IntraPrep, Coulter Immunotech) and stained with FITC-conjugated anti-Bax (Clone 4F11, Immunotech, Marseille, France) and anti-Bcl-2 monoclonal antibodies (Coulter Immunotech). The antigen 7A6, an early marker for proapoptotic mitochondrial signaling, ${ }^{39}$ was detected by the PE-labeled monoclonal antibody Apo2.7 (Coulter Immunotech) in lamina propria cells permeabilized by $0.01 \% \mathrm{NaN}_{3}$ and $100 \mu \mathrm{g} / \mathrm{ml}$ digitonin (Sigma-Aldrich, Deisenhofen, Germany).}

Analysis of caspase activity. PBMC were incubated with the indicated amounts of CD44 antibodies as specified above. Caspase-3 proteolytic activity was assessed by the corresponding fluorometric protease assay (R\&D Systems Inc., Wiesbaden-Nordenstadt, Germany). Cell lysate was tested for protease activity by addition of a caspase-3-specific peptide that was conjugated to the color reporter molecule $p$-nitroanaline (pNA). Cleavage of the peptide releases the chromophore pNA, which can be measured spectrophotometrically at $405 \mathrm{~nm}$. Mononuclear cells from lamina propria were isolated and stimulated as described above in the presence of $10 \mu \mathrm{M}$ inhibitor caspase-9 (Z-LEHD-FMK) or a broad-spectrum caspase inhibitor (Z-VAD-FMK, all from Calbiochem, Darmstadt, Germany).

Detection of apoptotic protease activating factor (APAF-1). PBMC or LPMC were lysed in protein lysis buffer containing $10 \mathrm{mM}$ Tris- $\mathrm{HCl}, \mathrm{pH} 7.4,1 \mathrm{mM}$ EDTA, $0.1 \%$ SDS, $0.1 \%$ Triton X-100 and protease inhibitor mix (Roche, Germany). Equal amounts of protein, as determined by BCA Protein Assay Kit (Pierce, Rockford, IL, USA), were added to electrophoresis sample buffer (Roth, Karlsruhe, Germany). After boiling, samples were loaded onto a 10\% SDS-PAGE gel and electrophoretically separated. Proteins were transferred to PVDF membrane (Roth) and detection was performed with a blotting analysis system by Roth. Rabbit anti-APAF-1 (BD Pharmingen) was incubated $(1: 1000)$ for $90 \mathrm{~min}$ and detected with swine-anti-rabbitIgG-HRP conjugated ( $1: 1000$ dilution, DAKO) for another $90 \mathrm{~min}$. Roti-Lumen I \& II (Roth) was used for detection of chemoluminescence. To evaluate correct loading, antibodies were removed from membrane by incubation with stripping buffer $(62.5 \mathrm{mM}$ Tris- $\mathrm{HCl}, \mathrm{pH} 6.7,2 \% \mathrm{SDS}, 100 \mathrm{mM}$ 2-mercaptoethanol) for $60 \mathrm{~min}$ at $70^{\circ} \mathrm{C}$. After washing, rabbit-anti- $\beta$-actin antibody (ABCAM, Cambridge, UK) was incubated in a $1: 1000$ dilution for $90 \mathrm{~min}$ and after washing, for the second step again swine-antirabbit-lgG was used.

Isolation and stimulation of cells from mice and induction of apoptosis. Female BALB/c and MLR/pr mice, deficient for Fas, were purchased from the Bundesinstitut für Risikobewertung (Berlin, Germany) and housed under specific pathogen-free conditions in our animal facility. BALB/c CD44v7-deleted mice were bred in our animal facility. ${ }^{7}$ All animal experiments were performed in accordance with institutional, state, and federal guidelines. For isolation of spleen cells from mice, spleen tissue was passed through a sieve to obtain single cell suspension. Erythrocytes were lysed in a buffer containing $10 \mathrm{mM} \mathrm{KHCO}_{3}, 155 \mathrm{mM}$ $\mathrm{NH}_{4} \mathrm{Cl}$, and $0.1 \mathrm{mM}$ EDTA ( $\left.\mathrm{pH} 7.5\right)$. Then cells were washed two times and viability was determined by Trypan blue staining. Whole spleen cells were incubated for 3 days on plate-bound anti-CD3 $(3 \mu \mathrm{g} / \mathrm{ml}$, clone $2 \mathrm{C} 11)$ and soluble anti-CD28 $(1 \mu \mathrm{g} /$ $\mathrm{ml}$, clone 37.51 , both kindly provided by J Hoffmann). After 3 days, $10 \mu \mathrm{g} / \mathrm{ml}$ antiCD44V7 mAb (clone LN7.1, mouse anti-mouse lgG1) or an isotype control was added for further $20-24 \mathrm{~h}$. The apoptosis rate was determined by the method of Nicoletti et al. ${ }^{37}$

Statistical analysis. Statistical tests for significance of differences were carried out by either Wilcoxon rank or Student's t-tests depending on the parameter examined, using the STAT-View software (Macintosh, Wiesbaden, Germany). Statistical differences were considered to be significant at the $P \leqslant 0.05$ level.

Acknowledgements. This work has been generously supported by the Broad Medical Research Program (IBD-0079) (BW), the Ludwig Demling Grant from the Deutsche Colitis Crohn Vereinigung (DCCV) (BW), and the Deutsche Forschungsgemeinschaft (AS) (DFG STA 295/2-3).

1. Scholmerich J. Inflammatory bowel disease: Pandorra's box, present and future. Ann NY Acad Sci 2006; 1072: 365-378.

2. Monteleone G, Fina D, Caruso R, Pallone F. New mediators of immunity and inflammation in inflammatory bowel disease. Curr Opin Gastroenterol 2006; 22: 361-364.

3. MacDonald TT, Monteleone G. Overview of role of the immune system in the pathogenesis of inflammatory bowel disease. Adv Exp Med Biol 2006; 579: 98-107.

4. Peppelenbosch MP, van Deventer SJ. T cell apoptosis and inflammatory bowel disease. Gut 2006; 53: 1556-1558.

5. Itoh J, De la Motte C, Strong SA, Levine AD, Fiocchi C. Decreased Bax expression by mucosal T cells favours resistance to apoptosis in Crohn's disease. Gut 2001; 49: 35-41.

6. Atreya R, Mudter J, Finotto S, Mullberg J, Jostock T, Wirtz S et al. Blockade of interleukin 6 trans signaling suppresses T-cell resistance against apoptosis in chronic intestinal inflammation: evidence in crohn disease and experimental colitis in vivo. Nat Med 2000; 6: 583-588.

7. Wittig BM, Johansson B, Zöller M, Schwärzler C, Günthert U. Abrogation of experimental colitis correlates with increased apoptosis in mice deficient for CD44v7. J Exp Med 2000; 191: 2053-2063.

8. Tiede I, Fritz G, Strand S, Poppe D, Dvorsky R, Strand D et al. CD28-dependent Rac1 activation is the molecular target of azathioprine in primary human CD4+ T lymphocytes. $J$ Clin Invest 2003; 111: 1133-1145.

9. Doering J, Begue B, Lentze MJ, Rieux-Laucat F, Goulet O, Schmitz J et al. Induction of T lymphocyte apoptosis by sulphasalazine in patients with Crohn's disease. Gut 2004; 53 : $1632-1638$.

10. ten Hove T, van Montfrans C, Peppelenbosch MP, van Deventer SJ. Infliximab treatment induces apoptosis of lamina propria T lymphocytes in Crohn's disease. Gut 2002; 50: 206-211.

11. Van den Brande JM, Koehler T, Zelinkova Z, Bennink RJ, Te Velde AA, Ten Kate F et al. Prediction of anti-TNF clinical efficacy by real-time visualisation of apoptosis in patients with Crohn's disease. Gut 2007; 56: 509-517.

12. Ponta $H$, Sherman L, Herrlich PA. CD44: from adhesion molecules to signalling regulators. Nat Rev Mol Cell Biol 2003; 4: 33-45.

13. Wittig B, Seiter S, Föger N, Schwärzler C, Günthert U, Zöller M. Functional activity of murine CD44 variant isoforms in allergic and delayed type hypersensitivity. Immunol Lett 1997; 57: 217-223.

14. Wittig B, Schwärzler C, Föhr N, Günthert U, Zöller M. Curative treatment of an experimentally induced colitis by a CD44 variant v7-specific antibody. J Immunol 1998; 161: 1069-1073.

15. Farkas $\mathrm{S}$, Hornung $\mathrm{M}$, Sattler $\mathrm{C}$, Anthuber M, Günthert $\mathrm{U}$, Herfarth $\mathrm{H}$ et al. Short-term treatment with anti-CD44v7 antibody, but not CD44v4, restores the gut mucosa in established chronic dextran sulfate sodium (DSS)-induced colitis in mice. Clin Exp Immunol 2005; 142: 260-267. 
16. Wittig BM, Stallmach A, Zeitz M, Günthert U. Functional involvement of CD44 variant 7 in gut immune response. Pathobiology 2002-2003; 70: 184-189.

17. Garcia de Tena J, Manzano L, Leal JC, San Antonio E, Sualdea V, Alvarez-Mon M. Distinctive pattern of cytokine production and adhesion molecule expression in peripheral blood memory CD4+ T cells from patients with active Crohn's disease. J Clin Immunol 2006; 26: 233-242.

18. Cella M, Facchetti F, Lanzavecchia A, Colonna M. Plasmacytoid dendritic cells activated by influenza virus and CD4OL drive a potent TH1 polarization. Nat Immunol 2000; 1: 305-310.

19. Fuss IJ, Neurath N, Boirivant M, Klein JS, de la Motte C, Strong SA. Disparate CD4 lamina propria lymphokine secretion profiles in inflammatory bowel disease. Crohn's disease LP cells manifest increased secretion of IFN-gamma, whereas ulcerative colitis LP cells manifest increased secretion of IL-5. J Immunol 1996; 157: 1261-1270.

20. Mielgo A, van Driel M, Bloem A, Landmann L, Günthert U. A novel antiapoptotic mechanism based on interference of Fas signaling by CD44 variant isoforms. Cell Death Differ 2006; 13: 465-477.

21. Bates RC, Edwards NS, Burns GF, Fisher DE. A CD44 survival pathway triggers chemoresistance via lyn kinase and phosphoinositide 3-kinase/Akt in colon carcinoma cells. Cancer Res 2001; 61: 5275-5283.

22. Henke C, Bitterman P, Roongta U, Ingbar D, Polunovsky V. Induction of fibroblast apoptosis by anti-CD44 antibody: implications for the treatment of fibropoliverative lung disease. Am J Pathol 1996; 149: 1639-1650.

23. Marhaba R, Zöller M. CD44 in cancer progression: adhesion, migration and growth regulation. J Mol Histol 2004; 35: 211-231.

24. Stauder R, Günthert U. CD44 isoforms: impact on lymphocyte activation and differentiation. Immunologist 1995; 3: 78-83.

25. Chakraborty G, Jain S, Behera R, Ahmed M, Sharma P, Kumar V et al. The multifaceted roles of osteopontin in cell signaling, tumor progression and angiogenesis. Curr Mol Med 2006; 6: 819-830

26. Danial NN, Korsmeyer SJ. Cell death: critical control points. Cell Death Differ 2004; 116 205-219.

27. Reed JC, Kroemer G. Mechanisms of mitochondrial membrane permeabilization Cell Death Differ 2000; 7: 1145
28. Steinman $L$. A brief history of $T(H) 17$, the first major revision in the $T(H) 1 / T(H) 2$ hypothesis of T cell-mediated tissue damage. Nat Methods 2007; 13: 139-145.

29. Zöller M, McElwee KJ, Vitacolonna M, Hoffmann R. Apoptosis resistance in peripheral blood lymphocytes of alopecia areata patients. J Autoimmun 2004; 23: 241-256.

30. Marhaba R, Freyschmidt-Paul P, Zöller M. In vivo CD44-CD49d complex formation in autoimmune disease has consequences on $\mathrm{T}$ cell activation and apoptosis resistance. Eur J Immunol 2006; 36: 3017-3032.

31. Wittig B, Seiter S, Schmidt DS, Zuber M, Neurath M, Zöller M. Selective upregulation of CD44 variant isoforms on peripheral blood leukocytes in patients with chronic inflammtory bowel disease and other systemic autoimmune diseases. Lab Invest 1999; 79 : 747-759.

32. Hart AL, Al-Hassi HO, Rigby RJ, Bell SJ, Emmanuel AV, Knight SC et al. Characteristics of intestinal dendritic cells in inflammatory bowel diseases. Gastroenterology 2005; 129: 50-65.

33. Leon F, Smythies LE, Smith PD, Kelsall BL. Involvement of dendritic cells in the pathogenesis of inflammatory bowel disease. Adv Exp Med Biol 2006; 579: 117-132.

34. Burkly LC. CD40 pathway blockade as an approach to immunotherapy. Adv Exp Med Biol 2001; 489: 135-152.

35. Firan $M$, Dhillon S, Estess $\mathrm{P}$, Siegelman $\mathrm{MH}$. Suppressor activity and potency among regulatory $T$ cells is discriminated by functionally active CD44. Blood 2006; 15: 619-627.

36. Lugering A, Lebiedz P, Koch S, Kucharzik T. Apoptosis as as therapeutic tool in IBD? Ann NY Acad Sci 2006; 1072: 62-77.

37. Nicoletti I, Migliorati G, Pagliacci MC, Grignani F, Riccardi C. A rapid and simple method for measuring thymocyte apoptosis by propidium iodide staining and flow cytometry. $\mathrm{J}$ Immunol Methods 1991; 139: 271-279.

38. Jonuleit $H$, Kuhn U, Muller G, Steinbrink K, Paragnik L, Schmitt E et al. Pro-inflammatory cytokines and prostaglandins induce maturation of potent immunostimulatory dendritic cells under fetal calf serum-free conditions. Eur J Immunol 1997; 12: 3135-3142.

39. Nagahara $Y$, Tanaka M, Shinomiya T. Mechanism of mitochondrial $7 A 6$ antigen exposure triggered by distinct apoptotic pathways: involvement of caspases. Cytometry A 2007; 71: 232-241. 